\title{
Linkage Disequilibrium and Physical Mapping of Pasl in Mice
}

\author{
Giacomo Manenti, ${ }^{1}$ Amanda Stafford, ${ }^{2}$ Laura De Gregorio, ${ }^{1}$ Manuela Gariboldi, ${ }^{1}$ \\ F. Stefania Falvella, ${ }^{1}$ Philip Avner, ${ }^{2}$ and Tommaso A. Dragani ${ }^{1,3}$ \\ ${ }^{1}$ Department of Experimental Oncology, Istituto Nazionale Tumori, Milan, Italy; ${ }^{2}$ Unite de Genetique Moleculaire Murine, \\ Unite de Recherche Associe Centre National de la Recherche Scientifique (URA CNRS) 1968, Institut Pasteur, Paris, France
}

\begin{abstract}
By using linkage disequilibrium (LD) analysis in 21 strains of known susceptibility to lung cancer and by assembling a YAC contig, we mapped to a $\sim 1.5-\mathrm{Mb}$ region on distal mouse chromosome 6 the Pasl locus, the major determinant of lung cancer predisposition in mice. Our results, on the basis of haplotype and phenetic analysis, suggest that the Pasls susceptibility allele is shared by several mouse-inbred strains of independent origin, which show either high or intermediate predisposition to lung tumorigenesis. Therefore, the Pas/ $I^{\mathrm{s}}$ allele is probably derived from an ancestral mouse rather than from independent mutations of the same gene. We showed the feasibility of LD in common inbred strains for the fine mapping of disease loci, and provided the biological basis and the reagents for the cloning of the Pasl gene.
\end{abstract}

The A/J mouse strain is highly susceptible to lung tumorigenesis and we have previously mapped the Pulmonary adenoma susceptibility 1 (Pas1) locus affecting inherited predisposition to lung tumorigenesis in this strain, to the distal region of chromosome 6 (Gariboldi et al. 1993). Subsequently, independent studies with the A/J strain have confirmed the major role of Pas1 in mouse lung tumorigenesis and have also supported the fact that the quantitative trait locus (QTL) peak for Pas1 is localized around Kras2 (Devereux et al. 1994; Festing et al. 1994; Manenti et al. 1995). As in many QTL studies, the linked region is too large $(>10 \mathrm{cM})$ for undertaking positional cloning of the causative gene. Approaches other than genetic linkage analysis should be devised to narrow the region.

Several strains of independent origin show high or intermediate susceptibility to lung tumorigenesis. Among these strains, we have obtained evidence recently that, in addition to the A/J strain, the SWR/J and the $\mathrm{BALB} / \mathrm{C}$ strains also carry the Pas $1^{\mathrm{s}}$ susceptibility allele, mapping close to the Kras2 locus (Manenti et al. 1997b). Strain polymorphisms at the Kras2 gene correlate with susceptibility to lung tumorigenesis (Malkinson and You 1994). On the basis of these observations, we hypothesize that the $\operatorname{Pas} 1^{\mathrm{s}}$ allele originated from a single founder and have designed a linkage disequilibrium (LD) study in mouse strains to test this hypothesis and to eventually narrow the candidate region for Pas1.

LD may be defined as the nonrandom association of marker alleles, usually mapping within a short chromosomal region, with a phenotype. Genetic linkage analysis is based only on recombination events that

\footnotetext{
${ }^{3}$ Corresponding author.
}

E-MAIL dragani@istitutotumori.mi.it; FAX 39-02-2390642. occur in a specific cross. In contrast, LD patterns rely on recombinations that have occurred over generations starting from the origin of the mutated allele in the founder mouse to the fixation of the mutation at homozygosity during inbreeding. LD analysis may therefore provide access to the equivalent of millions of meioses. LD has been successfully used in humans for the precise location of disease loci and has proved to be an important tool for the positional cloning of several disease genes (de la Chapelle 1993; Jorde 1995). The power of LD analysis for the precise mapping of a disease locus is especially clear in isolated populations, in which the disease-causing mutation originated from a single founder (Jorde 1995).

In experimental systems for the analysis of polygenic inheritance, disease loci have been mapped by genetic linkage studies typically carried out with a sample size of $<500$ meioses and haplotype analysis used to verify the candidacy of genes identified (Malo et al. 1994; MacPhee et al. 1995). To our knowledge, the potential of LD analysis for the fine mapping of disease genes has not, however, been tested in animals. If our hypothesis of a single founder of $\mathrm{Pas} 1^{\mathrm{s}}$ is correct, LD could be applied for the fine mapping of Pas1. Herein, we report that several markers located in the telomeric region of chromosome 6 exhibit significant LD with the genetic predisposition to lung cancer development. By combining LD and physical mapping, we shortened the Pas1 region to a $\sim 1.5-\mathrm{Mb}$ interval, between D6Mit57 and D6Mit304 markers. These results should make feasible the positional cloning of Pas1.

\section{RESULTS}

The genetic markers used are listed in Table 1. During our analysis, we observed several discrepancies be- 
Manenti et al.

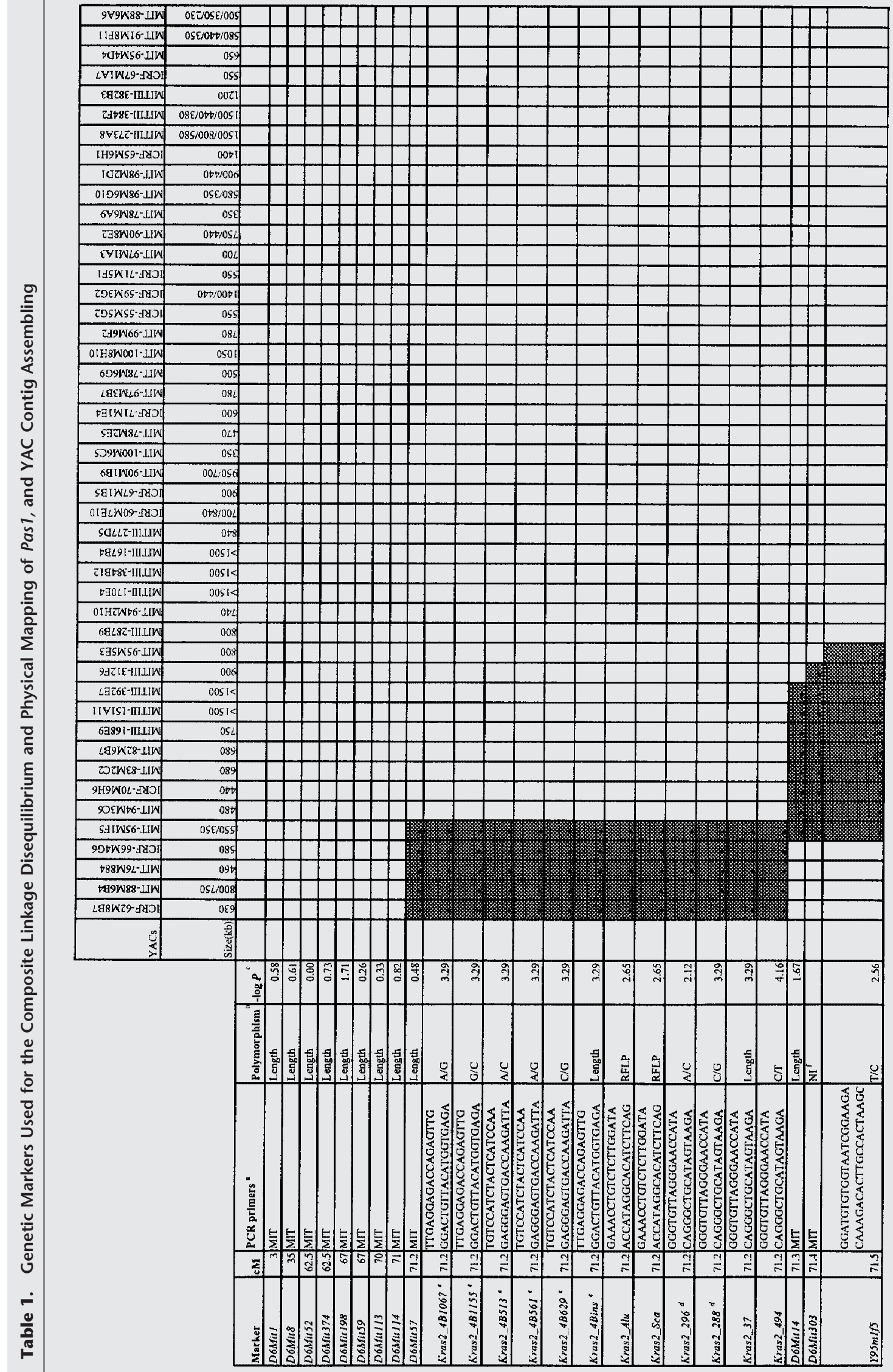




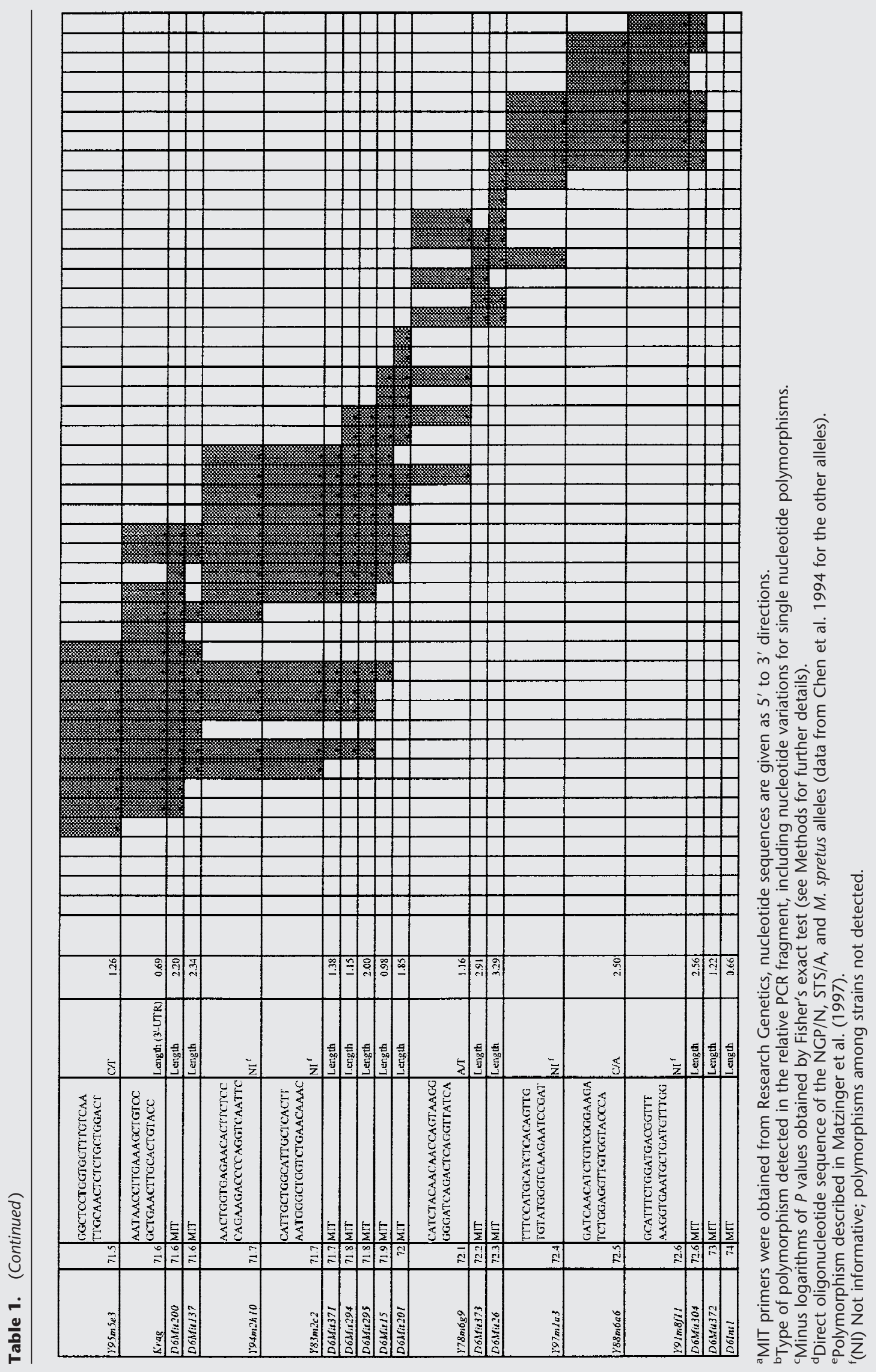

Genome Research 641 
tween the strain allele size of SSLPs reported by MIT and the PCR fragment length we obtained from our own analysis. Fifteen of 22 SSLPs (68\%) showed small differences in the allele size of at least one of the strains analyzed (data not shown). In the case of D6Mit26, for example, we found that the BALB/c allele is $196 \mathrm{bp}$ long instead of null, and the size of the Mus spretus allele is $202 \mathrm{bp}$ instead of the reported size of $200 \mathrm{bp}$. When these errors for D6Mit26 were corrected, all of the investigated strains fell into two classes that correlated well with susceptibility and resistance to lung tumor development, as indicated by the statistically significant association $(-\log P=3.29 ;-\log P$ being defined as the negative value of the logarithm of the $P$ value of the test statistic). Both the highly susceptible and the intermediate susceptible strains showed a common allele of $196 \mathrm{bp}$, whereas the resistant strains shared the same 202-bp-long allele (Table 2).

A YAC contig was constructed across the candidate region to provide reagents for the eventual cloning of the Pas1 gene. The D6Mit markers that localized in this interval (Dietrich et al. 1996) were screened across the mouse YAC libraries. Continuity of coverage required the addition of new STS markers obtained by YAC endclone isolation (Table 1). New markers lying within the contig were identified, including a 36-bp repeat polymorphism at Krag 3'-UTR, corresponding to nucleotide
4011-4046 in the GenBank sequence (accession no. MM02487). The strains carried either one or three repeats (data not shown). Strain polymorphisms detected in the YAC end-clone sequences yielded additional markers for LD analysis. A minimum set of five YACs of average size $840 \mathrm{~kb}$ is required to cover the D6Mit57-D6Mit304 interval. The size of this contig can be estimated at $1.5-2 \mathrm{Mb}$ if an average overlap of $50 \%$ between YAC clones is assumed. The order of markers across the YAC contig was in agreement with existing genetic maps. The one exception was D6Mit15, placed in the most telomeric group of markers on the MIT genetic map (Dietrich et al. 1996) but more proximally on the YAC contig as well as on EUCIB and MGD genetic maps (Mouse Genome Database 1998; Rhodes et al. 1998). A combined genetic and physical map of the region was assembled, with D6Mit57 $(71 \mathrm{cM})$ and D6Mit304 $(73 \mathrm{cM})$ markers as loci to anchor the genetic and physical maps. With the aim of defining genetic distance between markers in the contig, we attributed a 0.1-cM distance interval to markers separated by partially overlapping YAC clones (Table 1; Fig. 1).

A significant LD was found for markers extending across the whole YAC contig. Contingency tables with strains grouped into susceptible, intermediate, and resistance phenotypes resulted in the same LD pattern of two levels of tables (susceptible/intermediate and resis-

Table 2. Mouse Strains and Haplotypes at Genetic Markers Mapping in the Pas 1 Region

\begin{tabular}{|c|c|c|c|c|c|c|c|}
\hline \multirow[b]{2}{*}{ Strain } & \multirow[b]{2}{*}{ Susceptibility ${ }^{a}$} & \multicolumn{5}{|c|}{ Haplotype at selected markers ${ }^{b}$} & \multirow[b]{2}{*}{$\begin{array}{l}\text { Putative } \\
\text { Pas1 allele }\end{array}$} \\
\hline & & Kras2_494 & Kras2_37 & $\begin{array}{l}\text { Kras2_4B561 } \\
\text { Kras2_4B1067 }\end{array}$ & $Y 95 m 1 f 5$ & D6Mit26 & \\
\hline AKR/J & $\mathrm{R}$ & $\mathrm{T}$ & 2 & G & C & 202 & $r$ \\
\hline M. spretus & $\mathrm{R}$ & $\mathrm{T}$ & 1 & A & C & 202 & $r$ \\
\hline $\mathrm{C} 3 \mathrm{H} / \mathrm{HeJ}$ & $\mathrm{R}$ & $\mathrm{T}$ & 2 & G & C & 202 & $r$ \\
\hline C57L/J & $\mathrm{R}$ & $\mathrm{T}$ & 2 & G & $\mathrm{T}$ & 202 & $r$ \\
\hline C57BL/6J & $\mathrm{R}$ & T & 2 & G & $\mathrm{T}$ & 202 & $r$ \\
\hline SJL/J & $\mathrm{R}$ & T & 2 & G & C & 202 & $r$ \\
\hline DBA/2J & $\mathrm{R}$ & $\mathrm{T}$ & 2 & G & C & 202 & $r$ \\
\hline $\mathrm{SM} / \mathrm{J}$ & $\mathrm{R}$ & C & 1 & A & $\mathrm{T}$ & 196 & $s$ \\
\hline CBA/J & । & $\mathrm{C}$ & 1 & $A$ & $\mathrm{~T}$ & 196 & s \\
\hline LP/I & 1 & C & 1 & A & $\mathrm{T}$ & 196 & s \\
\hline $\mathrm{PL} / \mathrm{J}$ & I & C & 1 & A & $\mathrm{T}$ & 202 & $?$ \\
\hline $\mathrm{RF} / \mathrm{J}$ & I & C & 1 & $A$ & $\mathrm{~T}$ & 196 & $s$ \\
\hline $129 / \mathrm{SvJ}$ & 1 & C & 1 & A & $\mathrm{T}$ & 196 & s \\
\hline ST/bJ & I & C & 1 & A & $\mathrm{T}$ & 196 & $s$ \\
\hline $\mathrm{BALB} / \mathrm{c}$ & 1 & C & 1 & A & $\mathrm{T}$ & 196 & $s$ \\
\hline MA/Myl & 1 & C & 1 & A & $\mathrm{T}$ & 196 & $s$ \\
\hline STS/A & $S$ & C & 1 & A & $\mathrm{T}$ & 196 & $s$ \\
\hline $\mathrm{O} 20 / \mathrm{A}$ & $S$ & C & 1 & A & $\mathrm{T}$ & 196 & $s$ \\
\hline SWR/J & $S$ & C & 1 & A & $\mathrm{T}$ & 196 & s \\
\hline$A / J$ & S & C & 1 & A & $\mathrm{T}$ & 196 & $s$ \\
\hline $\mathrm{NGP} / \mathrm{N}$ & $S$ & C & 1 & $A$ & $\mathrm{~T}$ & 196 & s \\
\hline
\end{tabular}

a(R) Resistant; (I) Intermediate; (S) susceptible (see text for further details).

${ }^{b}$ Number of repeats at Kras2_37 marker, allele size for D6Mit26, and single nucletide polymorphisms for the other markers (see Table 2 and text for further details).

"Based on haplotype sharing of marker loci in LD with phenotype. 


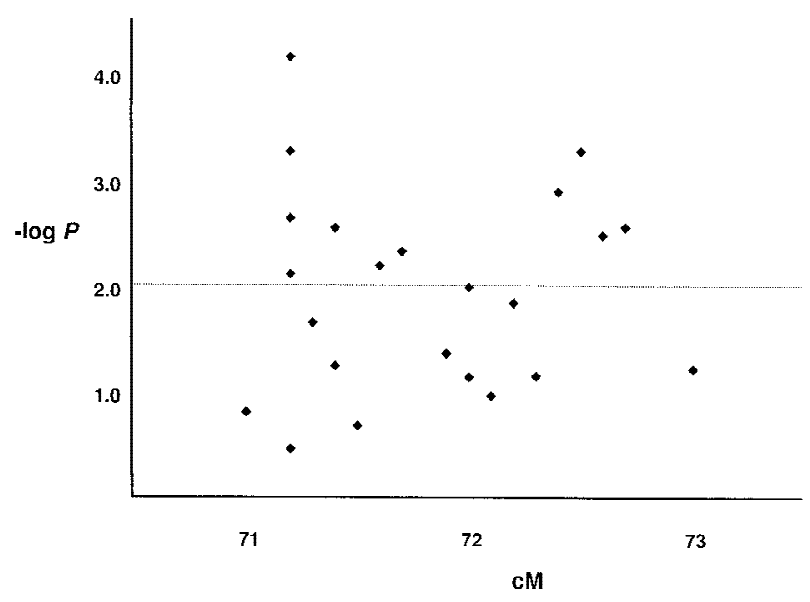

Figure 1 Composite genetic and physical map of the telomeric region of mouse chromosome 6 for LD mapping of the Pas 1 locus. Statistical significance of LD between marker polymorphisms and phenotype was estimated by Fisher's exact test and expressed as negative logarithm of $P$ values $(-\log P)$. The dotted line represents the $P=0.01(-\log P=2)$ cutoff significance value. Each point represents either a single genetic marker or multiple markers in the same position with the same $-\log P$ value (Table 1).

tant), although the statistical significance values were lower because of the increased degrees of freedom (data not shown). Analysis of variance by lung tumor multiplicity data gave the same LD pattern with significance values similar to those herein reported (data not shown). A total of 12 markers were typed in and around the Kras 2 locus, a short genomic region extending over $<40 \mathrm{~kb}$. In this region we found markers showing borderline significant LD (e.g. Kras2_296) as well as markers with a highly significant LD, such as the Kras2_494 polymorphism that showed the highest LD ( $-\log P=4.16)$ (Table 1). The D6Mit26 marker, located distally in the contig, showed the second highest LD value $(-\log P=3.29$ ) (Table 1$)$. Several other markers, located between the proximal and distal ends of the contig, were also associated with a significant LD $(-\log P>2)$ (Table 1; Fig. 1). We tested 10 additional genetic markers in chromosomal regions other than that of the distal part of chromosome 6. None of these markers showed significant LD with lung tumor susceptibility (data not shown).

Haplotype analysis with markers showing the most significant LD indicated that strains 129/SvJ, A/J, BALB/cJ, CBA/J, LP/J, MA/MyJ, NGP/J, O20/A, RF/J, ST/ bJ, STS/A, SM/J, and SWR/J carried the same haplotype (Table 2). Most of these strains show a high or intermediate predisposition to lung tumorigenesis (Malkinson 1989). Strain PL/J shared an identical haplotype to the above strains, except for the D6Mit26 marker (Table 2). On the other hand, the AKR/J, C3H/HeJ, C57BL/6J, C57L/J, DBA/2J, M. spretus, and SJL/J strains, which are resistant to lung tumorigenesis, showed a variable haplotype. Parsimony analysis of discrete state data, as well as the results of distance matrix programs, essentially produced the same phenetic tree (Fig. 2; data not shown). Two main branches, in addition to a branch containing only the $M$. spretus strain, were clearly separated. One of the main branches contained the strains with the high or intermediate predisposition to lung cancer, whereas the other branch contained the resistant strains.

\section{DISCUSSION}

We performed LD analysis in mouse strains of known susceptibility and resistance to lung tumorigenesis, using marker alleles around the Pas 1 locus. The use of LD analysis is based on the assumption that recombination is the major force determining the presence or absence of LD. Because one would expect to find genomic regions identical by descent in inbred strains, LD analysis in strains with a known phenotype may allow testing of whether the same locus is responsible for that given phenotype. LD is maintained only along

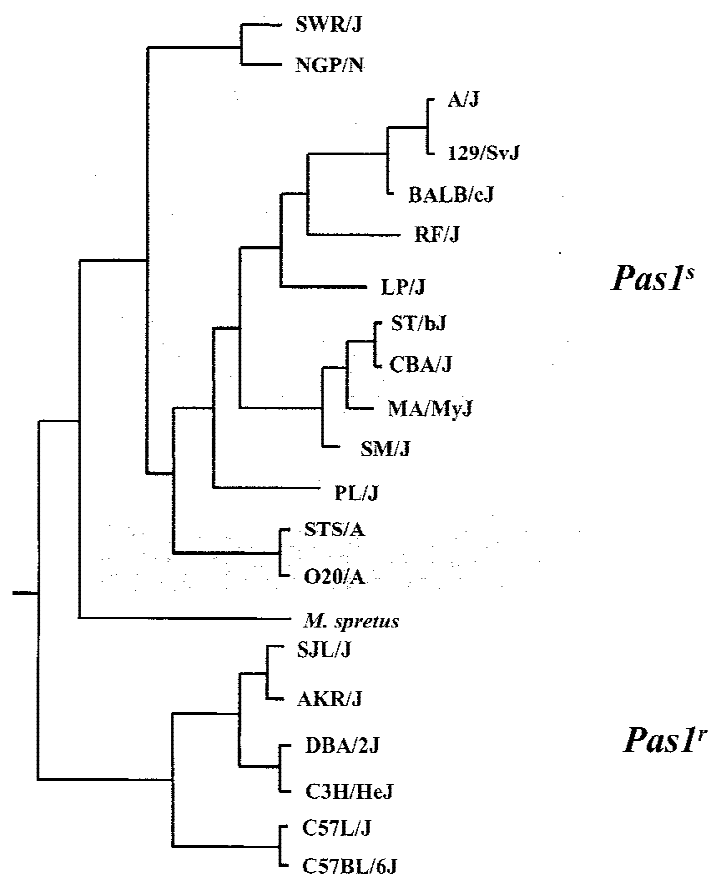

Figure 2 Phenetic tree of a 21-mouse inbred strain of known susceptibility to lung carcinogenesis, obtained by the FitchMargoliash distance matrix method, with marker polymorphisms mapped in the YAC contig containing the Pas 1 gene. The tree represents the estimated relationships among the strains for that short chromosomal region $(\sim 1.5 \mathrm{Mb})$, and it therefore provides an estimation of the phylogenetic origin of the $\operatorname{Pas} 7^{\mathrm{s}}$ allele. The gray box contains the putative Pas $7^{5}$ mice, which include the A/J, SWR/J, and BALB/C strains proved by genetic linkage to carry the Pas $1^{\mathrm{s}}$ allele. Also, the putative Pas $1^{\mathrm{r}}$ strains include the $\mathrm{C} 57 \mathrm{BL} / 6 \mathrm{~J}$, $\mathrm{C} 3 \mathrm{H} / \mathrm{HeJ}$, and $M$. spretus mice, established by genetic linkage studies as Pas $1^{\mathrm{r}}$ strains. 
short chromosomal regions, and it could therefore represent a new approach for the fine mapping of polygenic traits in mice.

However, several phenomena, including homoplasy and mutations, may have an important role in the maintenance or loss of LD. These confounding phenomena may explain why we found, within the same region, marker alleles showing a highly significant LD as well as marker alleles that were not in LD with the phenotypic trait (Fig. 1). As this is expected in LD studies, it is necessary to type many markers in LD studies to avoid false-positive and false-negative results. We suggest that the significance of LD analysis can only be assessed after comprehensive analysis of the whole region under investigation. We should also be aware that the prerequisite to limit LD mapping to those strains with a known phenotype (21 in our case) can weaken the confidence of any positive association, with some danger of type- 1 error. However, combining LD mapping with conventional cross-linkage data (as we partially did, as 6 of 21 strains carry a known Pas 1 allele), we could restrict the mapping of disease genes to a 1- to 2-cM region, suitable for positional cloning.

Our results indicate that inbred strains resistant to lung tumorigenesis show variations in their marker alleles. In contrast, all inbred strains susceptible or partially susceptible to lung tumorigenesis belonged to a single haplotype group (Table 2). Phenetic analysis confirmed the haplotype analysis by separating two branches of putative Pas $1^{\mathrm{s}}$ and Pas ${ }^{\mathrm{r}}$ strains (Fig. 2). Phenetic analysis formed new phylogenetic branches that were unrelated to the known historical origin of these strains (Festing 1993) and different from phylogenetic trees published previously (Fitch and Atchley 1985; Atchley and Fitch 1991) (Fig. 2). For example, the $\mathrm{C} 3 \mathrm{H} / \mathrm{He}$ (resistant) and the $\mathrm{CBA} / \mathrm{J}$ (intermediate) strains that derived from a single cross of a Bagg female and a DBA male (Festing 1993) are clearly separated in our analysis, whereas they are phylogenetically closely related (Atchley and Fitch 1991). The reason for this discrepancy is due to the selection for the genetic markers that lie within the short chromosomal region under examination. In this context, strain clustering suggests an origin of this small region from a common ancestor. Whereas homoplasy at microsatellite loci might also produce such strain clustering (Garza and Freimer 1996; Orti et al. 1997), the results we obtained with microsatellite-length polymorphisms were highly concordant with those derived from the analysis of single-nucleotide polymorphisms. Putative Pas $1^{\mathrm{s}}$ and Pas $1^{\mathrm{r}}$ groups of mice are separable (Fig. 2).

As was reported previously, strains A/J, SWR/J, and BALB/c carry the same Pas $1^{\mathrm{s}}$ allele (Manenti et al. 1997b); therefore, we can now infer that all strains sharing the same haplotype for these markers also carry the Pas $1^{\text {s }}$ allele (Table 2). A discrepancy is repre- sented by the $\mathrm{SM} / \mathrm{J}$ strain that carries the same haplotype as the putative Pas $1^{\mathrm{s}}$ animals, even though it is resistant to lung tumorigenesis (Malkinson 1989). However, the SM/J strain carries the Par1 and Par3 loci (Abujiang et al. 1997), which may inhibit phenotypic expression of the putative Pas $1^{\mathrm{s}}$ allele.

Putative Pas $1^{\mathrm{s}}$ strains are of varied geographical derivation (e.g., $\mathrm{A} / \mathrm{J}$ is United States derived and SWR/J strain is Swiss derived) and have originated at different times (e.g., A/J originated $>70$ years ago, whereas the $\mathrm{NGP} / \mathrm{N}$ is of a much more recent derivation; Festing 1993). The finding of an identical haplotype in such strains would seem to indicate that the Pas $1^{\mathrm{s}}$ susceptibility allele probably derived from an ancestral mouse, rather than originating in different strains as independent mutations. The presence of the Pas $1^{\text {s }}$ allele in several strains would indicate a relatively high frequency of this allele in the genus Mus. Genetic linkage experiments with crosses involving individual putative Pas ${ }^{\mathrm{s}}$ strains with a known Pas $^{\mathrm{r}}$ strain (e.g., C3H/HeJ, C57BL/6J, M. spretus) (Gariboldi et al. 1993; Devereux et al. 1994; Festing et al. 1994; Manenti et al. 1995) may allow us to verify whether this prediction is correct. However, the phenotypic expression of Pas $1^{\mathrm{s}}$ allele is reduced in most of these strains by the lung cancer modifier loci (Par loci) as we reported for the BALB/c strain (Dragani and Manenti 1997; Manenti et al. 1997c). This can explain why some Pas $1^{\mathrm{s}}$ strains (e.g., A/J, SWR/J, NGP/N) are highly susceptible, whereas most of the other Pas $1^{\mathrm{s}}$ strains show an intermediate susceptibility to lung tumorigenesis. The Par loci might therefore have an essential role in phenotypic expression of the inherited predisposition to lung cancer. Their identification and cloning should be further influenced by our results. The Par genes may represent interesting new targets for prospective lung cancer chemoprevention and therapeutic strategies (Dragani and Manenti 1997).

Our results provide information for the identification of the Pas1 gene. Both the fine physical mapping with YAC clones of the region containing the Pas1 gene and the identification of putative $\operatorname{Pas} 1^{\mathrm{s}}$ strains with haplotype analysis are relevant elements for subsequent strain comparison of germ-line variations of candidate Pas1 genes. The Pas1 gene may be located in the central region of the contig that shows significant LD. However, because the markers showing the best LD (Kras2_494 and D6Mit26) are positioned at the ends of the contig, we cannot rule out that two Pas1 genes might exist, located close to each of these two markers. Our approach cannot distinguish whether strains have both trait loci rather than one. Because the Pas $1^{\text {s }}$ allele is common in mouse strains, it may be predicted that it is also common in other species, including humans. Results that we have obtained in humans are in agreement with our present ones, because we have found a 
significant association of polymorphisms at KRAS2 and PTHLH loci (ends of the homologous human contig) with risk and prognosis for lung adenocarcinoma (Manenti et al. 1997a).

\section{METHODS}

\section{Mouse DNAs and Genetic Markers}

Genomic DNA samples were obtained from Jackson Laboratories (Bar Harbor, ME) $(129 / \mathrm{SvJ}, \mathrm{A} / \mathrm{J}, \mathrm{AKR} / \mathrm{J}, \mathrm{BALB} / \mathrm{cJ}, \mathrm{C} 3 \mathrm{H} /$ HeJ, C57BL/6J, C57L/J, CBA/J, DBA/2J, LP/J, MA/MyJ, M. spretus, PL/J, RF/J, SJL/J, SM/J, ST/bJ, SWR/J) or were kindly provided by Dr. I. Nakashima (Nagoya University, Japan; O20/A), Dr. M. Nishimura (Hamamatsu University, Japan; STS/A), and Dr. M. Mandel (National Cancer Institute, Bethesda, MD; NGP/N).

PCR primers for single nucleotide polymorphisms (SNPs) and for simple sequence length polymorphisms (SSLPs) are as reported in Table 1 . For SNP markers, aliquots of PCR reactions were loaded on an agarose gel to check the size and amount of amplified fragments. The remaining PCR mix was denatured in $0.4 \mathrm{M} \mathrm{NaOH} / 25 \mathrm{~mm}$ EDTA at room temperature and spotted onto nylon membrane. Allele-specific 15-mer oligonucleotides empassing the SNP were 5'- end-labeled with $\left[\gamma^{32} \mathrm{P}\right] \mathrm{dATP}(3000 \mathrm{Ci} / \mathrm{mm})$ (Amersham, Branchburg, NY) and T4 polynucleotide kinase (New England Biolabs, Beverly, MA). Allele-specific oligonucleotide (ASO) hybridizations were performed in tetramethylammonium chloride (TMAC) as reported (Manenti et al. 1994). SSLP markers were typed with PCR primers obtained from Research Genetics (Huntsville, $\mathrm{AL})$ and 25 radioactive PCR cycles $\left(55^{\circ} \mathrm{C}\right.$ annealing temperature); results were scored on $6 \%$ denaturing polyacrylamide gels. As markers to measure SSLPs length, we loaded in a single well the four ddNTPs-stopped samples of a radioactive sequencing reaction. The resulting 1-bp ladder was used to assign a precise length to the SSLP allele of each strain.

\section{YAC Library Screening}

The ICRF and MITII mouse YAC libraries (Larin et al. 1991; Kusumi et al. 1993) were screened by PCR. This involved screening a total of 49 super pools followed by a further 24reaction per positive super pools to identify the clone address. PCR reactions were carried out on a custom built Waffle Iron PCR machine with 35 cycles of $30 \mathrm{sec}$ at $94^{\circ} \mathrm{C} / 30 \mathrm{sec}$ at $55^{\circ} \mathrm{C} /$ $30 \mathrm{sec}$ at $72^{\circ} \mathrm{C}$. PCR products were visualized on $2 \%$ agarose gels. YAC clones from the MITIII library (Haldi et al. 1996) were obtained from Research Genetics on the basis of the coordinates provided on MIT's public access database. The individual YAC clones identified by these two strategies were checked for STS content by PCR screening with all STSs in the region to exclude false-positive and false-negative results that may occur in high-throughput YAC library screening. YAC clones were grown from frozen library stocks on AHC plates as unpurified well aliquots in AHC media. Agarose-embedded yeast DNA was loaded onto a $1 \%$ agarose gel and electrophoresed in $0.5 \times \mathrm{TBE}$ at $14^{\circ} \mathrm{C}$ on a Bio-Rad CHEF DRII-pulsed field gel apparatus with the following program: ramped switch time $50-110 \mathrm{sec}$ over $24 \mathrm{hr}$ at $200 \mathrm{~V}$. The DNA was transferred to a nylon membrane (Hybond $\mathrm{N}^{+}$, Amersham) by Southern blotting and hybridized with a radioactive probe prepared from $100 \mathrm{ng}$ of mouse cot1 DNA (GIBCO-BRL) labeled with Amersham's Megaprime DNA Labeling System with $\left[\alpha-{ }^{32} \mathrm{P}\right] \mathrm{dCTP}(\mathrm{ICN})$. The membrane was washed to a stringency of $0.1 \times \mathrm{SSC}$ at $65^{\circ} \mathrm{C}$ for $20 \mathrm{~min}$ and exposed for autoradiography.

\section{YAC End Clone Isolation and Polymorphism Detection}

YAC end clones were isolated by Vectorette, following the protocol of Riley et al. (1990) with YAC DNA embedded in blocks and digested with the restriction enzymes AluI and $R s a I$. The Vectorette products were gel purified and sequenced by cycle sequencing with fluorescence-labeled dideoxynucleotides with ABI's dideoxy Taq F.S. kit and electrophoresed on an ABI 377 automated sequencer. This sequence was used to derive PCR primers that were used to amplify YAC end clone sequences in four mouse strains, A/J, SWR/J, C57BL6/J and $\mathrm{C} 3 \mathrm{H} / \mathrm{HeJ}$. The amplified fragments were cloned into the pCR2.1 vector (Invitrogen, San Diego, CA) and at least two clones for each fragment were sequenced in both orientations on an ABI 377 sequencer. Sequences obtained from different strains were compared. When base differences were found among the four strains, ASOs were synthesized and hybridized to all mouse strains under investigation (Table 1).

\section{Linkage Disequilibrium Analysis and Phylogenetic Analysis}

As susceptibility to lung tumorigenesis, mouse strains were placed into two phenotype groups: The first group contained resistant strains and the second group contained susceptible and intermediate strains, according to published classification (Table 2) (van der Valk 1981; Malkinson 1989; Manenti et al. 1995). Linkage disequilibrium between strain segregation of marker alleles and lung tumor susceptibility was evaluated by Fisher's exact test. $P$ values were transformed in their negative logarithms, and a significant LD was considered if the $-\log$ $P>2(P<0.01)$.

Phenetic analysis of genetic elements surrounding the Pas1 locus was used to estimate phylogeny for this locus. In the region spanning the D6Mit113 to D6Int1 markers, we characterized 33 genetic markers (16 SSLPs, 12 SNPs, 2 RFLPs, and 3 small deletion/insertion) in 21 inbred strains with known susceptibility to lung tumor development. For the whole data set, a triangular matrix with the percentages of genotype differences was calculated by pairwise comparison of the strains (Canzian 1997). Among the 33 markers, 19 showed a binary state in all the inbred strains analyzed. The data set of discrete characters was first boot strapped and then used to estimate phylogeny according to Wagner and Dollo parsimony methods with MIX and DOLLOP programs, respectively (Fitch and Margoliash 1967; Canzian 1997; Farris 1997), with the PHYLYP package.

\section{ACKNOWLEDGMENTS}

We thank Dr. Federico Canzian for his suggestions, comments, and criticism. This work was partially funded by grants from Finalized Project Consiglio Nazionale Ricerche "Applicazioni Cliniche Ricerca Oncologica" and from the Associazione and Fondazione Italiana Ricerca Cancro (AIRC and FIRC) of Italy. Amanda Stafford was in receipt of a Wellcome Prize Travelling Research Fellowship. P.A. and A.S. were supported by grants from the GREG and the Juvenile Diabetes Foundation International.

The publication costs of this article were defrayed in part by payment of page charges. This article must therefore be 
hereby marked "advertisement" in accordance with 18 USC section 1734 solely to indicate this fact.

\section{REFERENCES}

Abujiang, P., M. Nishimura, T. Kamoto, K. Ichioka, M. Stato, and H. Hiai. 1997. Genetic resistance to urethane induced pulmonary adenomas in SMXA RI strains. Cancer Res. 57: 2904-2908.

Atchley, W.R. and W.M. Fitch, 1991. Gene trees and the origins of inbred strains of mice. Science 254: 554-558.

Canzian, F. 1997. Phylogenetics of the laboratory rat Rattus norvegicus. Genome Res. 7: 262-267.

Chen, B., L. Johanson, J.S. Wiest, M.W. Anderson, and M. You. 1994. The second intron of the K-ras gene contains regulatory elements associated with mouse lung tumor susceptibility. Proc. Natl. Acad. Sci. 91: 1589- 1593.

de la Chapelle, A. 1993. Disease gene mapping in isolated human populations: The example of Finland. J. Med. Genet. 30: 857-865.

Devereux, T.R., R.W. Wiseman, N. Kaplan, S. Garren, J.F. Foley, C.M. White, C. Anna, M.A. Watson, A. Patel, S. Jarchow, R.R. Maronpot, and M.W. Anderson. 1994. Assignment of a locus for mouse lung tumor susceptibility to proximal chromosome 19. Mamm. Genome 5: 749-755.

Dietrich, W.F., J. Miller, R. Steen, M.A. Merchant, D. Damron-Boles, Z. Husain, R. Dredge, M.J. Daly, K.A. Ingalls, T.J. O'Connor, et al. 1996. A comprehensive genetic map of the mouse genome. Nature 380: 149-152.

Dragani, T.A. and G. Manenti. 1997. Mom1 leads the pack. Nat. Genet. 17: 7-8.

Farris, J.S. 1977. Phylogenetic analysis under Dollo's Law. Syst. Zool. 26: $77-88$.

Festing, M.F.W. 1993. Origins and characteristics of inbred strains of mice. Mouse Genome 91: 393-509.

Festing, M.F.W., A. Yang, and A.M. Malkinson. 1994. At least four genes and sex are associated with susceptibility to urethane-induced pulmonary adenomas in mice. Genet. Res. 64: $99-106$.

Fitch, W.M. and E. Margoliash. 1967. Construction of phylogenetic trees. Science 155: 279-284.

Fitch, W.M. and W.R. Atchley. 1985. Evolution in inbred strains of mice appears rapid. Science 228: 1169-1175.

Gariboldi, M., G. Manenti, F. Canzian, F.S. Falvella, M.T. Radice, M.A. Pierotti, G. Della Porta, G. Binelli, and T.A. Dragani. 1993. A major susceptibility locus to murine lung carcinogenesis maps on chromosome 6. Nat. Genet. 3: 132-136.

Garza, J.C. and N.B. Freimer. 1996. Homoplasy for size at microsatellite loci in humans and chimpanzees. Genome Res. 6: 211-217.

Haldi, M.L., C. Strickland, P. Lim, V. VanBerkel, X. Chen, D. Noya, J.R. Korenberg, Z. Husain, J. Miller, and E.S. Lander. 1996. A comprehensive large- insert yeast artificial chromosome library for physical mapping of the mouse genome. Mamm. Genome 7: 767-769.

Jorde, L.B. 1995. Linkage disequilibrium as a gene-mapping tool. Am. J. Hum. Genet. 56: 11-14.

Kusumi, K., J.S. Smith, J.A. Segre, D.S. Koos, and E.S. Lander. 1993. Construction of a large-insert yeast artificial chromosome library of the mouse genome. Mamm. Genome 4: 391-392.

Larin, Z., A.P. Monaco, and H. Lehrach. 1991. Yeast artificial chromosome libraries containing large inserts from mouse and human DNA. Proc. Natl. Acad. Sci. 88: 4123-4127.

MacPhee, M., K.P. Chepenik, R.A. Liddell, K.K. Nelson, L.D. Siracusa, and A.M. Buchberg. 1995. The secretory phospholipase A2 gene is a candidate for the Mom1 locus, a major modifier of $A p c^{M i n}$-induced intestinal neoplasia. Cell 81: 957-966.

Malkinson, A.M. 1989. The genetic basis of susceptibility to lung tumors in mice. Toxicology 54: 241-271.

Malkinson, A.M. and M. You. 1994. The intronic structure of cancer-related genes regulates susceptibility to cancer. Mol. Carcinog. 10: 61-65.

Malo, D., K. Vogan, S. Vidal, J. Hu, M. Cellier, E. Schurr, A. Fuks, N. Bumstead, K. Morgan, and P. Gros. 1994. Haplotype mapping and sequence analysis of the mouse Nramp gene predict susceptibility to infection with intracellular parasites. Genomics 23: 51-61.

Manenti, G., S. Pilotti, F.C. Re, G. Della Porta, and M.A. Pierotti. 1994. Selective activation of ras oncogenes in follicular and undifferentiated thyroid carcinomas. Eur. J. Cancer 30A: 987-993.

Manenti, G., F.S. Falvella, M. Gariboldi, T.A. Dragani, and M.A. Pierotti. 1995. Different susceptibility to lung tumorigenesis in mice with an identical Kras2 intron 2. Genomics 29: 438-444.

Manenti, G., L. De Gregorio, S. Pilotti, F.S. Falvella, M. Incarbone, F. Ravagnani, M.A. Pierotti, and T.A. Dragani, 1997a. Association of chromosome 12p genetic polymorphisms with lung adenocarcinoma risk and prognosis. Carcinogenesis 18: $1917-1920$

Manenti, G., M. Gariboldi, A. Fiorino, A.I. Zedda, M.A. Pierotti, and T.A. Dragani. 1997b. Pas1 is a common lung cancer susceptibility locus in three mouse strains. Mamm. Genome 8: 801-809.

Manenti, G., M. Gariboldi, A. Fiorino, N. Zanesi, M.A. Pierotti, and T.A. Dragani. 1997c. Genetic mapping of lung cancer modifier loci specifically affecting tumor initiation and progression. Cancer Res. 57: 4164- 4166.

Matzinger, S.A., B. Chen, Y. Wang, K.A. Crist, G.D. Stoner, G.J. Kelloff, RA, Lubet, and M. You. 1997. Tissue-specific expression of the K-ras allele from the A/J parent in (A/J x TSG-p53) F1 mice. Gene 188: 261-269.

Mouse Genome Database (MGD) 1998. Mouse genome informatics, The Jackson Laboratory, Bar Harbor, ME. (URL:http:// www.informatics.jax.org/).

Orti, G., D.E. Pearse, and J.C. Avise. 1997. Phylogenetic assessment of length variation at a microsatellite locus. Proc. Natl. Acad. Sci. 94: $10745-10749$.

Rhodes, M., R. Straw, S. Fernando, A. Evans, T. Lacey, A. Dearlove, J. Greystrong, J. Walker, P. Watson, P. Weston et al. 1998. A high-resolution microsatellite map of the mouse genome. Genome Res. 8: 531-542.

Riley, J., R. Butler, D. Ogilvie, R. Finniear, D. Jenner, S. Powell, R, Anand, J.C. Smith, and A.F. Markham. 1990. A novel, rapid method for the isolation of terminal sequences from yeast artificial chromosome (YAC) clones. Nucleic Acids Res. 18: $2887-2890$

van der Valk, M.A. 1981. Survival, tumor incidence and gross pathology in 33 mouse strains. In Mammary tumors in the mouse. (ed. J. Hilgers and M. Sluyser), pp. 46-115. Elsevier, Amsterdam, The Netherlands.

Received July 29, 1998; accepted in revised form May 26, 1999. 


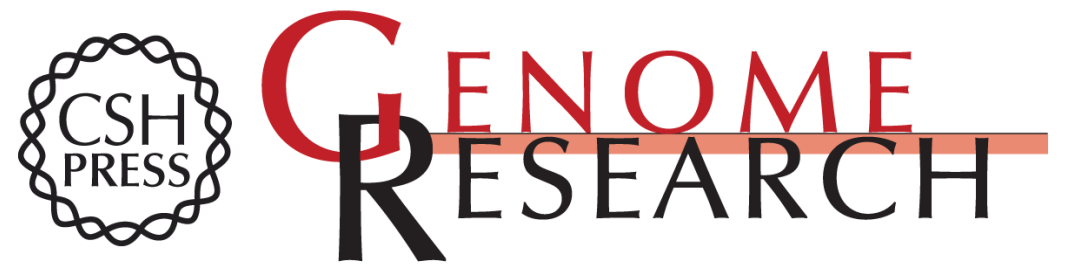

\section{Linkage Disequilibrium and Physical Mapping of Pas1 in Mice}

Giacomo Manenti, Amanda Stafford, Laura De Gregorio, et al.

Genome Res. 1999 9: 639-646

Access the most recent version at doi:10.1101/gr.9.7.639

References This article cites 31 articles, 12 of which can be accessed free at:

http://genome.cshlp.org/content/9/7/639.full.html\#ref-list-1

\section{License}

Email Alerting Receive free email alerts when new articles cite this article - sign up in the box at the Service top right corner of the article or click here.

\section{Affordable, Accurate Sequencing.}

To subscribe to Genome Research go to: https://genome.cshlp.org/subscriptions 